\title{
EMITTANCE GROWTH FROM ROTATED QUADRUPOLES IN HEAVY ION ACCELERATORS
}

\author{
John J. Barnard \\ Lawrence Livernore National Laboratory, University of Califomia \\ P.O. Box 5508, L-440, Livernore California 94550, USA
}

This paper was prepared for submittal to the

Proceedings of the IEEE 1995 Particle Accelerator Conference

Dallas, Texas

May 1-5, 1995

May 2, 1995

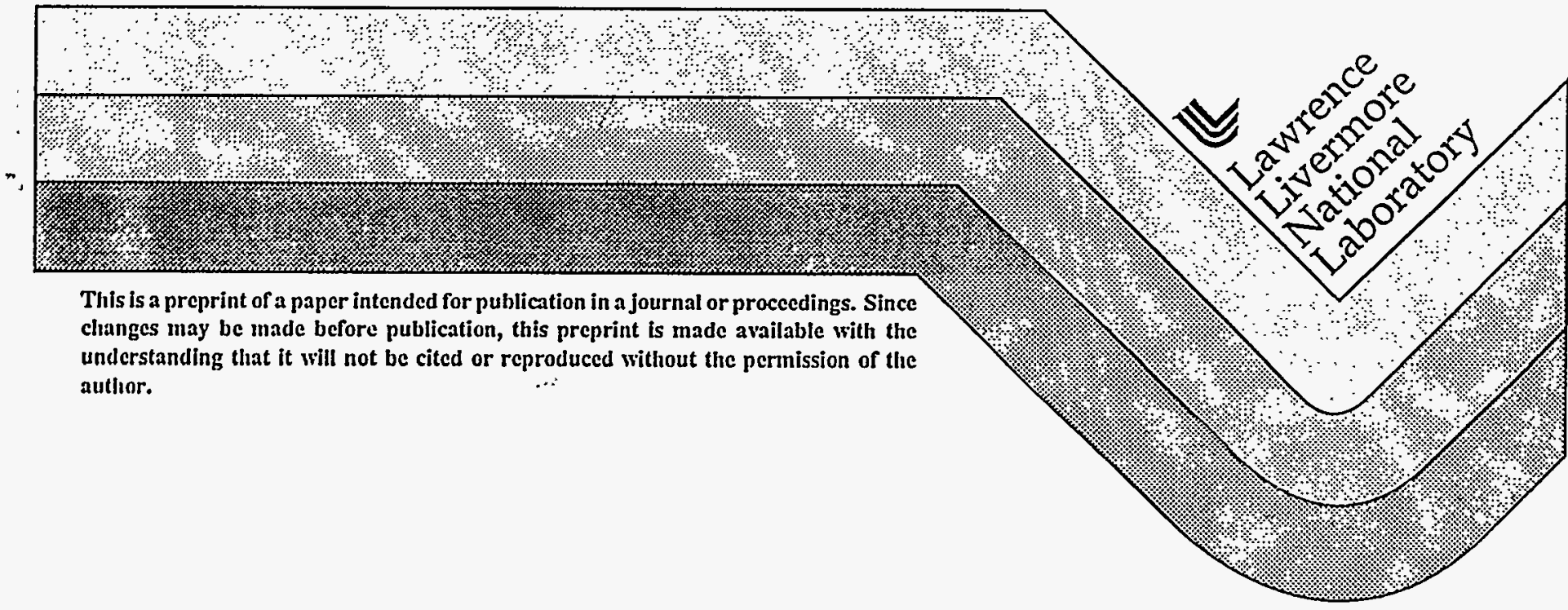




\section{DISCLAIMER}

This document was prepared as an account of worksponsored by an agency of the United States Government. Neither the United States Govemment nor the University of California nor any of their employees, makes any warranty, express or implied, or assumes any legal liability of responsibility for the accuracy, completeness, or usefulness of any information, apparatus, product, or process disclosed, or represents that its use would not infringe privately owned rights. Reference herein to any specific commercial products, process, or service by trade name, trademark, manufacturer, or otherwise, does not necessarily constitute or imply its endorsement, recommendation, or favoring by the United States Government or the University of California. The views and opinions of authors expressed herein do not necessarily state or reflect those of the United States Government or the University of Califomia, and shall not be used for advertising or product endorsement purposes. 


\section{Emittance Growth from Rotated Quadrupoles in Heavy Ion Accelerators*}

John J. Barnard

Lawrence Livermore National Laboratory, L-440, Livermore, CA 94550

\begin{abstract}
We derive a set of moment equations which incorporates linear quadrupolar focusing and space-charge defocusing, in the presence of rotational misalignments of the quadrupoles about the direction of beam propagation. Although the usual beam emittance measured relative to fixed transverse $x$ and $y$ coordinate axes is not constant, a conserved emittance-like quantity has been found. Implications for alignment tolerances in accelerators for heavyion inertial fusion are discussed.
\end{abstract}

\section{INTRODUCTION}

One class of misalignments of interest to accelerator designers is that class characterized by a rotation of the beam optical elements about the axis of propagation. Rotated dipoles, for example, are known to cause the centroid of a particle beam to wander off axis, (since the rotations will result in momentum impulses in the positive and negative $y$ [vertical] direction.) However rotated quadrupoles will not cause an initially aligned beam centroid to become misaligned. Quadrupole rotations do however, create a linear coupling between the two transverse directions, $x$ (horizontal) and $y$, in the equations of motion. Since this coupling enters linearly in the equations of motion, individual particle oscillation frequencies can be shifted, and this has implications for resonance avoidance in synchrotrons, (see ref. [1] and references therein). In this paper, we are interested in the effects on the emittance of beams with non-negligible space charge, such as those proposed for heavy ion inertial fusion.

We derive a set of moment equations which incorporates this coupling, and which serves as a generalization to the conventional envelope equations. We show that even when the equations of motion are linear in $x$ and $y$, the beam emittance measured relative to fixed $x$ and $y$ coordinate axes is not constant, although a conserved emittancelike quantity can be defired. If not corrected, a beam will acquire a finite angular momentum and rotation angle, before passing through a final focusing lens, thereby limiting the achievable final spot size. The results presented here will be of use in determining alignment tolerances in heavy ion accelerators.

\section{EQUATIONS OF MOTION}

To obtain an estimate, we assume that the force on an ion comes from two sources only: The external focusing from a purely quadrupolar field, and the space charge of the beam (image forces have been neglected). For the purposes of this calculation we assume that the space charge is distributed in a uniform density ellipse, but we allow the semi-axes and the rotation angle of the ellipse to evolve as a function of the axial coordinate $z$. We assume that a quadrupole is rotated by an angle $\theta$ from the $x$-axis, and that the beam is rotated by an angle $\alpha$ from the $x$-axis.

"Work performed under the auspices of the U.S. D.O.E. by LLNL under contract W-7405-ENG-48
The relation between the coordinates in the quadrupole frame (indicated by subscript 0 ) and the lab frame (no subscript) are given by:

$$
x=x_{0} \cos \theta-y_{0} \sin \theta ; \quad y=y_{0} \cos \theta+x_{0} \sin \theta
$$

Similarly, the relation between the coordinates in the rotated beam frame (in which the beam semi-axes are parallel to the coordinate axes and are indicated by subscript b) and the lab frame are given by:

$$
x-\langle x\rangle=x_{b} \cos \alpha-y_{b} \sin \alpha ; \quad y-\langle y\rangle=y_{b} \cos \alpha+x_{b} \sin \alpha
$$

Here () indicates a statistical average over the distribution function. For a non-relativistic beam moving at constant velocity $\beta c$ along the $z$ axis, the paraxial equations of motion can then be written as:

$$
\begin{aligned}
& x^{\prime \prime}=K_{q x x} x+K_{q x y} y+K_{s x x}(x-\langle x\rangle)+K_{s x y}(y-\langle y\rangle) \\
& y^{\prime \prime}=K_{q y y} y+K_{q y x} x+K_{s y y}(y-\langle y\rangle)+K_{s y x}(x-\langle x\rangle)
\end{aligned}
$$

Here primes ( $\left.{ }^{\prime}\right)$ indicate derivatives with respect to $z$ and

$$
\begin{aligned}
& K_{q x x} \equiv K_{q x 0} \cos ^{2} \theta+K_{q y 0} \sin ^{2} \theta=K_{q x 0} \cos 2 \theta \\
& K_{q x y} \equiv\left(K_{q x 0}-K_{q y 0}\right)(\sin \theta \cos \theta)=K_{q x 0} \sin 2 \theta \\
& K_{q y x} \equiv\left(K_{q x 0}-K_{q y 0}\right)(\sin \theta \cos \theta)=K_{q x y} \\
& K_{q y y} \equiv K_{q y 0} \cos ^{2} \theta+K_{q x 0} \sin ^{2} \theta=-K_{q x 0} \cos 2 \theta \\
& K_{s x x} \equiv K_{s x b} \cos ^{2} \alpha+K_{s y b} \sin ^{2} \alpha \\
& K_{s x y} \equiv\left(K_{s x b}-K_{s y b}\right)(\sin \alpha \cos \alpha) \\
& K_{s y x} \equiv\left(K_{s x b}-K_{s y b}\right)(\sin \alpha \cos \alpha)=K_{s x y} \\
& K_{s y y} \equiv K_{s y b} \cos ^{2} \alpha+K_{s x b} \sin ^{2} \alpha
\end{aligned}
$$

and where

$$
\begin{aligned}
K_{q x 0} & \equiv \pm \frac{B^{\prime}}{[B \rho]} \text { or } \frac{E^{\prime}}{\beta c[B \rho]} ; \quad K_{q y 0} \equiv-K_{q x 0} \\
K_{s x b} & \equiv K /\left[2\left(\Delta x_{b}^{2}+\left(\Delta x_{b}^{2} \Delta y_{b}^{2}\right)^{1 / 2}\right)\right] \\
K_{s y b} & \equiv K /\left[2\left(\Delta y_{b}^{2}+\left(\Delta x_{b}^{2} \Delta y_{b}^{2}\right)^{1 / 2}\right)\right]
\end{aligned}
$$

and $\Delta x_{b}^{2}$ and $\Delta y_{b}^{2}$ are the moments in the rotated beam frame:

$$
\begin{aligned}
& \Delta x_{b}^{2}=\Delta x^{2} \cos ^{2} \alpha+\Delta y^{2} \sin ^{2} \alpha+2 \Delta x y \cos \alpha \sin \alpha \\
& \Delta y_{b}^{2}=\Delta y^{2} \cos ^{2} \alpha+\Delta x^{2} \sin ^{2} \alpha-2 \Delta x y \cos \alpha \sin \alpha
\end{aligned}
$$

Here, $K \equiv 2 q I /\left(\beta^{3} A I_{o}\right)$ is the perveance, $q$ is the charge state of the ions, $A$ is the atomic mass of the ions, $\beta$ is the velocity of the ions in units of $c, I_{0} \equiv 4 \pi \epsilon_{0} m_{p} c^{3} / e$ is the proton characteristic current ( $\cong 31 \mathrm{MA}), I$ is the ion beam current, $B^{\prime}$ and $E^{\prime}$ are quadrupole magnetic or electric field gradients respectively, $[B \rho] \equiv A m_{p} \beta c / q e$ is the ion rigidity, $m_{p}$ is the proton mass, $e$ is the proton charge, $\epsilon_{0}$ is the free space permittivity and the operator $\Delta$ is defined (as in ref. [2]) by $\Delta a b=\langle a b\rangle-\langle a\rangle\langle b\rangle$ (e.g. $\Delta x^{2} \equiv\left\langle x^{2}\right\rangle-\langle x\rangle^{2}$ ), where () indicates average over particles. 
Note that the space-charge force, is just the force obtained from the potential of a uniform density ellipse (ref. [3]), but where the semi-axes $a$ and $b$ have been replaced by $2\left(\Delta x_{b}^{2}\right)^{1 / 2}$ and $2\left(\Delta y_{b}^{2}\right)^{1 / 2}$, respectively, and where the location of the centroid determines the zero point of the space-charge force.

The beam rotation angle $\alpha$ may be expressed in terms of second order moments. From eq. (2), $\Delta x^{2}-\Delta y^{2}=$ $\left(\Delta x_{b}^{2}-\Delta y_{b}^{2}\right) \cos 2 \alpha$ and $\Delta x y=(1 / 2)\left(\Delta x_{b}^{2}-\Delta y_{b}^{2}\right) \sin 2 \alpha$, so that

$$
\tan 2 \alpha=\frac{2 \Delta x y}{\Delta x^{2}-\Delta y^{2}}
$$

In deriving eq. 9 we have used the fact that $\Delta x y_{b}=0$.

\section{MOMENT EQUATIONS}

Let the distribution function $f$, be the number of particles $d N$ per unit transverse phase space volume,

$$
f\left(x, x^{\prime}, y, y^{\prime}, z\right)=\frac{\mathrm{d} N}{\mathrm{~d} x \mathrm{~d} x^{\prime} \mathrm{d} y \mathrm{~d} y^{\prime}}
$$

The evolution of $f$ is described by the Vlasov/Collisonless Boltzmann Equation:

$$
\frac{\partial f}{\partial z}+x^{\prime} \frac{\partial f}{\partial x}+x^{\prime \prime} \frac{\partial f}{\partial x^{\prime}}+y^{\prime} \frac{\partial f}{\partial y}+y^{\prime \prime} \frac{\partial f}{\partial y^{\prime}}=0
$$

where $x^{\prime \prime}$ and $y^{\prime \prime}$ are determined by the equations of motion (eqs. [3] and [4] ).

The average of a variable $\xi$ over the continuous distribution is given by:

$$
\langle\xi\rangle(z) \equiv \frac{1}{N} \iiint \int \xi f\left(x, x^{\prime}, y, y^{\prime}, z\right) \mathrm{d} x \mathrm{~d} x^{\prime} \mathrm{d} y \mathrm{~d} y^{\prime} .
$$

Using integration by parts, it is straightforward to calculate the evolution of the following second order moments:

$$
\begin{aligned}
& \frac{\mathrm{d} \Delta x^{2}}{\mathrm{~d} z}=2 \Delta x x^{\prime} \\
& \frac{\mathrm{d} \Delta x x^{\prime}}{\mathrm{d} z}=\Delta x^{\prime 2}+K_{x x} \Delta x^{2}+K_{x y} \Delta x y \\
& \frac{\mathrm{d} \Delta x^{\prime 2}}{\mathrm{~d} z}=2 K_{x x} \Delta x x^{\prime}+2 K_{x y} \Delta x^{\prime} y \\
& \frac{\mathrm{d} \Delta y^{2}}{\mathrm{~d} z}=2 \Delta y y^{\prime} \\
& \frac{\mathrm{d} \Delta y y^{\prime}}{\mathrm{d} z}=\Delta y^{\prime 2}+K_{y y} \Delta y^{2}+K_{y x} \Delta x y \\
& \frac{\mathrm{d} \Delta y^{\prime 2}}{\mathrm{~d} z}=2 K_{y y} \Delta y y^{\prime}+2 K_{y x} \Delta x y^{\prime} \\
& \frac{\mathrm{d} \Delta x y}{\mathrm{~d} z}=\Delta x^{\prime} y+\Delta x y^{\prime} \\
& \frac{\mathrm{d} \Delta x^{\prime} y}{\mathrm{~d} z}=\Delta x^{\prime} y^{\prime}+K_{x x} \Delta x y+K_{x y} \Delta y^{2} \\
& \frac{\mathrm{d} \Delta x y^{\prime}}{\mathrm{d} z}=\Delta x^{\prime} y^{\prime}+K_{y y} \Delta x y+K_{y x} \Delta x^{2} \\
& \frac{\mathrm{d} \Delta x^{\prime} y^{\prime}}{\mathrm{d} z}=K_{x x} \Delta x y^{\prime}+K_{x y} \Delta y y^{\prime}+ \\
& K_{y y} \Delta x^{\prime} y+K_{y x} \Delta x x^{\prime}
\end{aligned}
$$

Similarly the evolution of the first-order moments is given by:

$$
\begin{aligned}
& \frac{\mathrm{d}\langle x\rangle}{\mathrm{d} z}=\left\langle x^{\prime}\right\rangle \\
& \frac{\mathrm{d}(y)}{\mathrm{d} z}=\left\langle y^{\prime}\right\rangle \\
& \frac{\mathrm{d}\left\langle x^{\prime}\right\rangle}{\mathrm{d} z}=K_{q x x}\langle x\rangle+K_{q x y}\langle y\rangle \\
& \frac{\mathrm{d}\left(y^{\prime}\right\rangle}{\mathrm{d} z}=K_{q y y}\langle y\rangle+K_{q y x}\langle x\rangle
\end{aligned}
$$

In eq. 11 ,

$$
\begin{aligned}
& K_{x x} \equiv K_{q x x}+K_{s x x} \\
& K_{y y} \equiv K_{q y y}+K_{s y y} \\
& K_{x y} \equiv K_{q x y}+K_{s x y} \\
& K_{y x} \equiv K_{q y x}+K_{s y x}=K_{x y} .
\end{aligned}
$$

Note that the four equations for the first order moments depend only on first order moments, and the ten equations for the second order moments depend only on second order moments, so that each set of equations forms a closed set. The centroid motion is thus decoupled from the envelope motion calculated with respect to the centroid.

The rms emittances defined along the $x$ and $y$ lab frame axes are defined by:

$$
\begin{aligned}
& \epsilon_{x} \equiv 4\left(\Delta x^{2} \Delta x^{\prime 2}-\left(\Delta x x^{\prime}\right)^{2}\right)^{1 / 2} \\
& \epsilon_{y} \equiv 4\left(\Delta y^{2} \Delta y^{\prime 2}-\left(\Delta y y^{\prime}\right)^{2}\right)^{1 / 2}
\end{aligned}
$$

Using eqs. (11) we can calculate the derivatives of $\epsilon_{x}^{2}$ and $\epsilon_{y}^{2}$ (again assuming constant $\beta$ ):

$$
\begin{aligned}
& \frac{\mathrm{d} \epsilon_{x}^{2}}{\mathrm{~d} z}=32 K_{x y}\left(\Delta x^{2} \Delta x^{\prime} y-\Delta x y \Delta x x^{\prime}\right) \\
& \frac{\mathrm{d} \epsilon_{y}^{2}}{\mathrm{~d} z}=32 K_{y z}\left(\Delta y^{2} \Delta x y^{\prime}-\Delta x y \Delta y y^{\prime}\right)
\end{aligned}
$$

Since the rotated quadrupoles induce finite correlations between $x$ and $y$ the rms emittances are not conserved.

We may also define a quantity $l \equiv \Delta x y^{\prime}-\Delta x^{\prime} y$, which is proportional to the $z$ component of the angular momentum. Again using eqs. (11) and some manipulations involving the definitions, and eqs. (4), (5) and (9), we find,

$$
\frac{\mathrm{d} l}{\mathrm{~d} z}=\left(K_{q y y}-K_{q z x}\right) \Delta x y+K_{q z y}\left(\Delta x^{2}-\Delta y^{2}\right)
$$

As can be seen from eq. 14, the angular momentum is not necessarily conserved when the quadrupoles are rotated. Physically, after a beam has passed through a quadrupole the beam will in general be elliptical. On passing through a quadrupole rotated relative to the first, the principal axis of the elliptical beam will not align with the quadrupole axes and $a$ torque will be applied to the beam, causing a rotation of the beam. (Note also that eq. 14, does not depend on the self space-charge forces of the beam, as expected).

Because the focusing strength is a function of $z$, the effective external potential well within which the beam travels is $z$ dependent, and so the transverse beam energy $H$ is also not a constant in $z$. However, in the hard-edge model, within each quadrupole and drift section the focusing strength is assumed constant, and therefore the transverse energy is constant. We may use the result of ref. [2], adding the kinetic and potential energy terms to obtain a total transverse energy. To obtain the potential energy of the beam in the external quadrupole field, we transform $\Delta x_{0}^{2}$ and $\Delta y_{0}^{2}$ to the lab frame. The result is

$$
\begin{aligned}
2 H= & \Delta x^{\prime 2}+\Delta y^{\prime 2}- \\
& K_{q x 0}\left(\left(\Delta x^{2}-\Delta y^{2}\right) \cos 2 \theta+2 \Delta x y \sin 2 \theta\right)- \\
& K \ln \left(\left(\Delta x_{b}^{2}\right)^{1 / 2}+\left(\Delta y_{b}^{2}\right)^{1 / 2}\right)
\end{aligned}
$$


Here $\Delta x_{b}^{2}$ and $\Delta y_{b}^{2}$ may be expressed in laboratory quantities using eqs. (7) and (8).

\section{V. "EMITTANCE-LIKE" CONSTANT OF THE MOTION}

Although the emittance is not a constant with respect to $z$, a quantity which is related to the emittance is conserved. We define a generalized emittance $\epsilon_{g}$ by:

$$
\epsilon_{g}^{2} \equiv \frac{1}{2} \epsilon_{x}^{2}+\frac{1}{2} \epsilon_{y}^{2}+16\left(\Delta x y \Delta x^{\prime} y^{\prime}-\Delta x y^{\prime} \Delta x^{\prime} y\right)
$$

It is readily shown using eqs. 11 and 13 that $\frac{d \epsilon_{g}^{2}}{d z}=0$.

\section{EXAMPLES OF RESULTS}

A code was written to integrate eqs. (11). The results for $\epsilon_{x}$ and $\epsilon_{g}$ are plotted in figure 1 for a singly charged potassium beam $(A=39)$ with a current of $2 \mathrm{~mA}$, an energy of $80 \mathrm{kV}$, initial emittances $\epsilon_{x}=\epsilon_{y}=2.5 \times 10^{-5}$ $\mathrm{m} \mathrm{rad}, K_{q x o}=30.9 \mathrm{~m}^{-2}$, and with $\langle\theta\rangle=0.0234$ and $\Delta \theta^{2}=0.0156$. The occupancy of the quadrupoles was 0.33 and the half-lattice period was $0.36 \mathrm{~m}$. The integration length was 40 half-lattice periods.

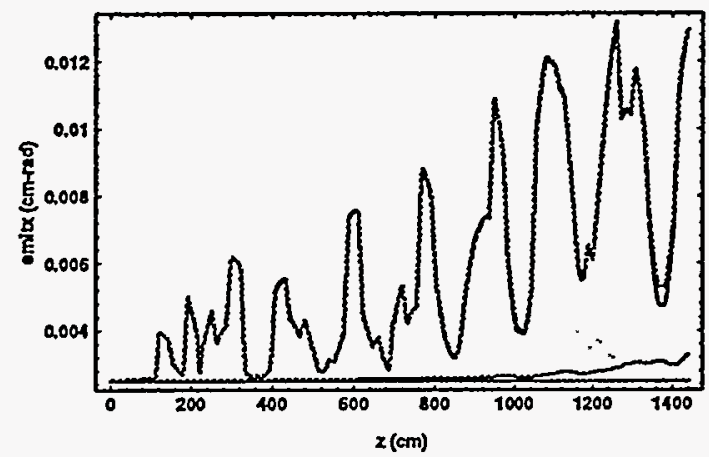

Figure 1. $\epsilon_{x}$ (oscillating) and $\epsilon_{g}$ (nearly constant) vs $z$ for both integration of eq. 11, and particle-in-cell results.

Also shown in Figure 1 is a 2D particle-in-cell (PIC) simulation with the same parameters, for an initial distribution that is KV (ref.[3]), propagating through a pipe with circular cross section and $6 \mathrm{~cm}$ radius. The near identical overlap of the curves suggests that if the initial distribution is KV the assumption that the space charge field remains linear is at least a good approximation and possibly an exact result. The small increase in $\epsilon_{g}$ for large $z$ is probably due to the non-linear image forces arising because of the finite pipe radius in the PIC simulations.

When these results are applied to the small recirculator of ref. [4], we find that with $2 \mathrm{mrad}$ rms errors, there occurs only a $2 \%$ increase in emittance for a beam which drifts (rather than accelerates) the nominal 15 laps. When the rotation errors are random over all 15 laps the emittance increases by about $50 \%$. An accelerated beam will presumably show behavior somewhere in-between. A generalization of the theory presented here to include acceleration is in progress.

\section{DISCUSSION AND CONCLUSION}

In inertial fusion applications, the ultimate goal is to focus the beam onto a small, $2-3 \mathrm{~mm}$ spot at the target.
The final emittance is one of the important parameters needed to calculate the achievable final spot size (see e.g. ref.[5]). When quadrupole rotation errors are present, the beam will in general have a finite rotation angle and rotation rate, and will focus down to a more elliptical shape than in the absence of errors, reducing the power level that falls within a given spot radius. Analogous to the case of centroid displacements, it is conceivable that a system of intentionally rotated quadrupoles could compensate for the accumulated errors if the ten moments in eq. 11 are known.

In summary, we have used a formulation, in which the major assumption is that the space charge force can be calculated by assuming that the beam remains a uniform density ellipse with a shape that evolves in $z$. Under this assumption we have a derived a set of moment equations which generalizes the conventional envelope equations. We have found the misalignments cause the beam to acquire an overall angular momentum, and an increase in emittance measured relative to fixed laboratory axes. A generalized emittance has been constructed which is a conserved quantity (when the forces remain linear). Particle-in-cell results have shown agreement with the moment equations, and have suggested that the formulation may be exact if the initial distribution is KV. We have applied this method to estimate rotation alignment tolerances in the small recirculator of ref [4], and have suggested that this formulation will be useful when setting alignment tolerances and/or correction methods in an inertial fusion driver.

\section{ACKNOWLEDGEMENTS}

I wish to thank S. M. Lund for useful discussions and suggestions and for critically reading a draft version of this paper, and A. Friedman and W. M. Sharp for other useful comments.

\section{REFERENCES}

[1]. Edwards, D.A., and Syphers, M.J., "An Introduction to the Physics of High Energy Accelerators," [John Wiley and Sons, Inc., New York], Chapter 5, (1993).

[2]. J.J. Barnard, H.D. Shay, S.S. Yu, A. Friedman, and D.P. Grote, "Emittance Growth in Heavy Ion Recirculatọrs," Proceedings of the 1992 Linear Accelerator Conference, 1992 August 2428, Ottawa, Ontario, Canada, C.R. Hoffman, ed., .AECL 10728 (AECL Research, Chalk River, Canada) p. 229.

[3]. I.M. Kapchinsky and V.V. Vladimirskij, "Limitations of proton beam current in a strong focusing linear accelerator with the beam space charge," Proceedings of the International Conference on High Energy Accelerators, (CERN, Geneva, 1959), p. 274.

[4]. A. Friedman, J.J. Barnard, M. Cable, D.A. Callahan, F. Deadrick, D.P. Grote, H. Kirbie, D. Longinotti, S.M. Lund, L. Nattrass, M. Nelson, M. Newton, C. Sangster, W.M. Sharp, D. Judd, S.S. Yu, "Progress Toward a Prototype Recirculating Induction Accelerator for Heavy-Ion Fusion," these proceedings.

[5].E.P. Lee, "Accelerator and Final Focus Model for an Induction Linac Based HIF System Study," in Heavy Ion Inertial Fusion, AIP Conference Proceedings 152, M. Reiser, T. Godlove, and R. Bangerter, Eds. (AIP, NY,1986), p. 461. 
Technical Information Department . Lawrence Livermore National Laboratory University of California . Livermore, California 94551

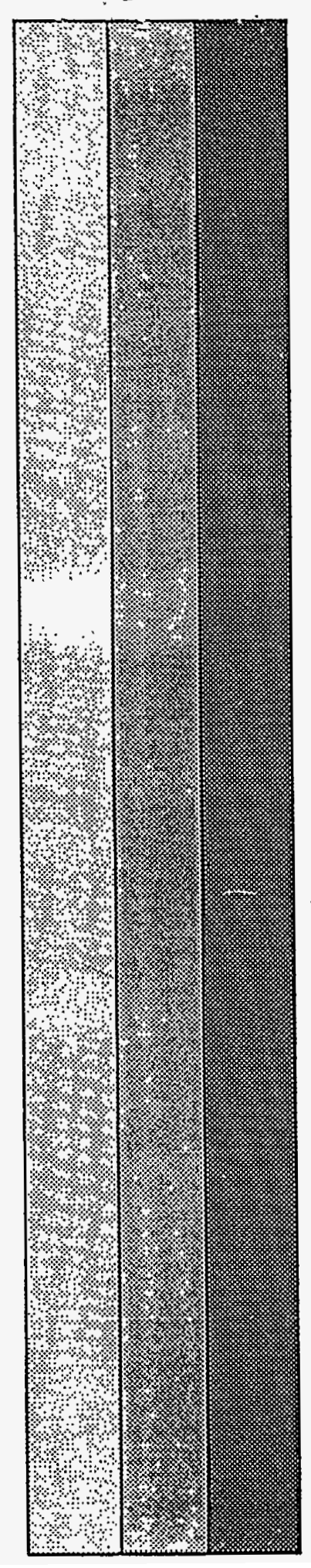

